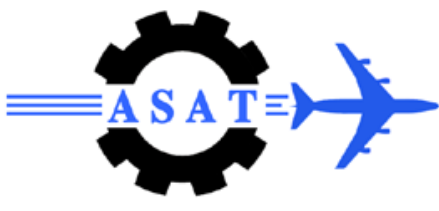

\title{
On Solution of Nonlinear Cubic Non-Homogeneous Schrodinger Equation
}

\author{
Magdy A. El-Tawil ${ }^{*}$, Sherif E. Nasr ${ }^{\dagger}$, H. El Zoheiry ${ }^{\ddagger}$
}

\begin{abstract}
In this paper, a perturbing nonlinear non-homogeneous Schrodinger equation is studied under limited time interval, complex initial conditions and zero Neumann conditions. The perturbation and Picard approximation methods together with the eigenfunction expansion and variational parameters methods are used to introduce an approximate solution for the perturbative nonlinear case for which a power series solution is proved to exist. Using Mathematica, the solution algorithm is tested through computing the possible orders of approximations. The method of solution is illustrated through case studies and figures.
\end{abstract}

Keywords: Nonlinear Schrodinger equation, Perturbation, Eigenfunction expansion, Mathematica, Picard Approximation.

\section{Introduction}

The nonlinear Schrodinger equation (NLS) is the principal equation to be analyzed and solved in many fields, see [1-5] for examples. The NLS equation arises in many application areas [6-8] such as wave propagation in nonlinear media, surface wave in sufficiently deep waters and signal propagation in optical fibers. The NLS is one of the most important models of mathematical physics arising in a great array of contexts $[9,10]$ as for conductor electronics, optics in nonlinear media, photonics, plasmas, fundamentation of quantum mechanics, dynamics of accelerators, mean-field theory of Bose-Einstein condensates or in biomolecule dynamics.

In the last ten decades, there are a lot of NLS problems depending on additive or multiplicative noise in the random case $[11,12]$ or a lot of solution methodologies in the deterministic case. Wang M. et al. [13] obtained the exact solutions to NLS using what they called the sub-equation method. They got four kinds of exact solutions of the equation for which no sign to the initial or boundary conditions type is made. Xu L. and Zhang J. [14] followed the same previous technique in solving the higher order NLS.

Sweilam N. [15] solved a nonlinear cubic Schrodinger equation which gives rise to solitary solutions using variational iteration method. Zhu S. [16] used the extended hyperbolic

\footnotetext{
* Engineering Mathematics Department, Faculty of Engineering, Cairo University, Giza, Egypt, magdyeltawil@yahoo.com .

${ }^{\dagger}$ Engineering Mathematics Department, Faculty of Engineering, Fayoum University, Fayoum, Egypt, sherif_eid96@yahoo.com .

‡ Engineering Mathematics Department, Faculty of Engineering, Cairo University, Giza, Egypt, hanafy_elzoheiry@yahoo.com .
} 
auxiliary equation method in getting the exact explicit solutions to the higher order NLS without any conditions. Sun J. et al. [17] solved an NLS with an initial condition using Lie group method. By using coupled amplitude phase formulation, Parsezian K. and Kalithasan B. [18] constructed the quartic anharmonic oscillator equation from the coupled higher order NLS. Two-dimensional grey solitons to the NLS were numerically analyzed by Sakaguchi H. and Higashiuchi T. [19]. The generalized derivative NLS was studied by Huang D. et al. [20] introducing a new auxiliary equation expansion method.

\section{The General Linear Case}

Consider the non homogeneous linear Schrodinger equation:

$i \frac{\partial u(t, z)}{\partial z}+\alpha \frac{\partial^{2} u(t, z)}{\partial t^{2}}=F_{1}(t, z)+i F_{2}(t, z),(t, z) \in(0, T) \times(0, \infty)$

where $u(t, z)$ is a complex valued function which is subjected to:

I.C.: $u(t, 0)=f_{1}(t)+i f_{2}(t)$, a complex valued function,

B. C.: $u(0, z)=u(T, z)=0$.

Let $u(t, z)=\psi(t, z)+i \phi(t, z), \psi, \phi$ are real valued functions. The following coupled equations are got as follows:

$\frac{\partial \phi(t, z)}{\partial z}=\alpha \frac{\partial^{2} \psi(t, z)}{\partial t^{2}}+G_{1}(t, z)$

$\frac{\partial \psi(t, z)}{\partial z}=\alpha \frac{\partial^{2} \phi(t, z)}{\partial t^{2}}+G_{2}(t, z)$

where $\psi(t, 0)=f_{1}(t), \phi(t, 0)=f_{2}(t), G_{1}(t, z)=-F_{1}(t, z), \quad G_{2}(t, z)=F_{2}(t, z)$, and all corresponding other I.C. and B.C. are zeros.

Eliminating one of the variables in equations (4) and (5), one can get the following independent equations:

$$
\begin{aligned}
& \frac{\partial^{4} \psi(t, z)}{\partial t^{4}}+\frac{1}{\alpha^{2}} \frac{\partial^{2} \psi(t, z)}{\partial t^{2}}=\frac{1}{\alpha^{2}} \tilde{\psi}_{1}(t, z), \\
& \frac{\partial^{4} \phi(t, z)}{\partial t^{4}}+\frac{1}{\alpha^{2}} \frac{\partial^{2} \phi(t, z)}{\partial t^{2}}=\frac{1}{\alpha^{2}} \tilde{\psi}_{2}(t, z),
\end{aligned}
$$

where

$$
\begin{aligned}
& \tilde{\psi}_{1}(t, z)=\frac{\partial G_{2}(t, z)}{\partial z}-\alpha \frac{\partial^{2} G_{1}(t, z)}{\partial t^{2}}, \\
& \tilde{\psi}_{2}(t, z)=\alpha \frac{\partial^{2} G_{2}(t, z)}{\partial t^{2}}+\frac{\partial G_{1}(t, z)}{\partial z}
\end{aligned}
$$


Using the eigenfunction expansion technique [24], the following solution expressions are obtained:

$\psi(t, z)=\sum_{n=0}^{\infty} T_{n}(z) \sin \left(\frac{n \pi}{T}\right) t$

$\phi(t, z)=\sum_{n=0}^{\infty} \tau_{n}(z) \sin \left(\frac{n \pi}{T}\right) t$

where $T_{n}(z)$ and $\tau_{n}(z)$ can be got through the applications of initial conditions and then solving the resultant second order differential equations using the method of variational of parameter [17]. The final expressions can be got as in the following:

$T_{n}(z)=\left(C_{1}+A_{1}(z)\right) \sin \beta_{n} z+\left(C_{2}+B_{1}(z)\right) \cos \beta_{n} z$

$\tau_{n}(z)=\left(C_{3}+A_{2}(z)\right) \sin \beta_{n} z+\left(C_{4}+B_{2}(z)\right) \cos \beta_{n} z$

where

$\beta_{n}=\alpha\left(\frac{n \pi}{T}\right)^{2}$

$A_{1}(z)=\frac{1}{\beta_{n}} \int \tilde{\psi}_{1 n}(z ; n) \sin \left(\beta_{n} z\right) d z$,

$B_{1}(z)=\frac{-1}{\beta_{n}} \int \tilde{\psi}_{1 n}(z ; n) \sin \left(\beta_{n} z\right) d z$,

$A_{2}(z)=\frac{1}{\beta_{n}} \int \tilde{\psi}_{2 n}(z ; n) \cos \left(\beta_{n} z\right) d z$,

$B_{2}(z)=\frac{-1}{\beta_{n}} \int \tilde{\psi}_{2 n}(z ; n) \cos \left(\beta_{n} z\right) d z$,

in which

$\tilde{\psi}_{1 n}(z ; n)=\frac{2}{T} \int \tilde{\psi}_{1}(t, z) \sin \left(\frac{n \pi}{T} t\right) d t$,
$\tilde{\psi}_{2 n}(z ; n)=\frac{2}{T} \int \tilde{\psi}_{2}(t, z) \sin \left(\frac{n \pi}{T} t\right) d t$,

The following conditions should also be satisfied:

$C_{2}=\frac{2}{T} \int_{0}^{T} f_{1}(t) \sin \left(\frac{n \pi}{T} t\right) d t-B_{1}(0)$,

$C_{4}=\frac{2}{T} \int_{0}^{T} f_{2}(t) \sin \left(\frac{n \pi}{T} t\right) d t-B_{2}(0)$. 
Finally the following solution is obtained:

$u(t, z)=\psi(t, z)+i \phi(t, z)$,

Or

$|u(t, z)|^{2}=\psi^{2}(t, z)+\phi^{2}(t, z)$.

\section{The Non-Linear Case}

Consider the non-homogeneous non-linear Schrodinger equation:

$$
\begin{gathered}
i \frac{\partial u(t, z)}{\partial z}+\alpha \frac{\partial^{2} u(t, z)}{\partial t^{2}}+\varepsilon|u(t, z)|^{2} u(t, z)+i \gamma u(t, z)=F_{1}(t, z)+i F_{2}(t, z), \quad(t, z) \\
\in(0, T) x(0, \infty)
\end{gathered}
$$

where $u(t, z)$ is a complex valued function which is subjected to the initial and boundary conditions mentioned before in equations (2), (3) respectively.

\section{Lemma [21-23]}

The solution of equation (25) with the constraints (2), (3) is a power series in $\varepsilon$ if exists.

\section{Proof}

at $\varepsilon=0$, the following linear non-homogenous equation is got:

$$
\begin{gathered}
\begin{array}{c}
i \frac{\partial u_{0}(t, z)}{\partial z}+\alpha \frac{\partial^{2} u_{0}(t, z)}{\partial t^{2}}+i \gamma u_{0}(t, z)=F_{1}(t, z)+i F_{2}(t, z) \\
(t, z) \in(0, T) x(0, \infty)
\end{array} \\
u_{0}(t, z)=\psi_{0}(t, z)+i \phi_{0}(t, z)
\end{gathered}
$$

where,

$$
\begin{aligned}
& \psi_{0}(t, z)=e^{-\gamma z} \sum_{n=0}^{\infty} T_{0 n}(z) \sin \left(\frac{n \pi}{T}\right) t \\
& \phi_{0}(t, z)=e^{-\gamma z} \sum_{n=0}^{\infty} \tau_{0 n}(z) \sin \left(\frac{n \pi}{T}\right) t
\end{aligned}
$$

where $T_{0 n}(z)$ and $\tau_{0 n}(z)$ can be calculated as the linear case equations (12), (13) respectively.

Following Pickard approximation, equation (25) can be rewritten as:

$$
\begin{aligned}
i \frac{\partial u_{n}(t, z)}{\partial z}+ & \alpha \frac{\partial^{2} u_{n}(t, z)}{\partial t^{2}}+i \gamma u_{n}(t, z) \\
& =F_{1}(t, z)+i F_{2}(t, z)-\varepsilon\left|u_{n-1}(t, z)\right|^{2} u_{n-1}(t, z), \quad n \geq 1
\end{aligned}
$$


at $n=1$, the iterative equation takes the form

$$
\begin{aligned}
i \frac{\partial u_{1}(t, z)}{\partial z}+ & \alpha \frac{\partial^{2} u_{1}(t, z)}{\partial t^{2}}+i \gamma u_{1}(t, z)=F_{1}(t, z)+i F_{2}(t, z)-\varepsilon\left|u_{0}(t, z)\right|^{2} u_{0}(t, z) \\
& =\varepsilon k_{1}(t, z)
\end{aligned}
$$

which can be solved as a linear case with zero initial and boundary conditions. The following general solution can be obtained:

$\psi_{1}(t, z)=e^{-\gamma z} \sum_{n=0}^{\infty}\left(T_{0 n}(z)+\varepsilon T_{1 n}(z)\right)(z) \sin \left(\frac{n \pi}{T}\right) t$

$\phi_{1}(t, z)=e^{-\gamma z} \sum_{n=0}^{\infty}\left(\tau_{0 n}(z)+\varepsilon \tau_{1 n}(z)\right)(z) \sin \left(\frac{n \pi}{T}\right) t$

$u_{1}(t, z)=\psi_{1}(t, z)+i \phi_{1}(t, z)$

$u_{1}(t, z)=u_{1}^{(0)}+\varepsilon u_{1}^{(1)}$,

At $n=2$, the following equation is obtained:

$$
\begin{aligned}
i \frac{\partial u_{2}(t, z)}{\partial z}+ & \alpha \frac{\partial^{2} u_{2}(t, z)}{\partial t^{2}}+i \gamma u_{2}(t, z)=F_{1}(t, z)+i F_{2}(t, z)-\varepsilon\left|u_{1}(t, z)\right|^{2} u_{1}(t, z) \\
& =\varepsilon k_{2}(t, z)
\end{aligned}
$$

which can be solved as a linear case with zero initial and boundary conditions. The following general solution can be obtained:

$u_{2}(t, z)=u_{2}{ }^{(0)}+\varepsilon u_{2}^{(1)}+\varepsilon^{2} u_{2}^{(2)}+\varepsilon^{3} u_{2}^{(3)}+\varepsilon^{4} u_{2}^{(4)}$,

Continuing like this, one can get:

$u_{n}(t, z)=u_{n}{ }^{(0)}+\varepsilon u_{n}^{(1)}+\varepsilon^{2} u_{n}^{(2)}+\varepsilon^{3} u_{n}{ }^{(3)}+\cdots+\varepsilon^{(n+m)} u_{n}{ }^{(n+m)}$.

As $n \rightarrow \infty$, the solution (if exists) can be reached as $u(t, z)=\lim _{n \rightarrow \infty} u_{n}(t, z)$. Accordingly the solution is a power series in $\varepsilon$.

According to the previous lemma, one can assume the solution of equation (25) as the following:

$u(t, z)=\sum_{n=0}^{\infty} \varepsilon^{n} u_{n}(t, z)$

Let $u(t, z)=\psi(t, z)+i \phi(t, z), \psi, \phi$ : are real valued functions. The following coupled equations are got: 
$\frac{\partial \phi(t, z)}{\partial z}=\alpha \frac{\partial^{2} \psi(t, z)}{\partial t^{2}}+\varepsilon\left(\psi^{2}+\phi^{2}\right) \psi-\gamma \phi-F_{1}(t, z)$

$\frac{\partial \psi(t, z)}{\partial z}=-\alpha \frac{\partial^{2} \phi(t, z)}{\partial t^{2}}-\varepsilon\left(\psi^{2}+\phi^{2}\right) \phi-\gamma \psi+F_{2}(t, z)$,

where $\psi(t, 0)=f_{1}(t), \phi(t, 0)=f_{2}(t)$, and all corresponding other I.C. and B.C. are zeros.

As a perturbation solution, one can assume that the prototype equations to be solved are:

$\frac{\partial \phi_{i}(t, z)}{\partial z}=\alpha \frac{\partial^{2} \psi_{i}(t, z)}{\partial t^{2}}+G_{i}^{(1)}, \quad i \geq 1$

$\frac{\partial \psi_{i}(t, z)}{\partial z}=\alpha \frac{\partial^{2} \phi_{i}(t, z)}{\partial t^{2}}+G_{i}^{(2)}, \quad i \geq 1$

where $\psi_{i}(t, 0)=\delta_{i, 0} f_{1}(t), \phi_{i}(t, 0)=\delta_{i, 0} f_{2}(t)$, and all other corresponding initial conditions are zeros. $G_{i}^{(1)}, G_{i}^{(2)}$ are functions to be computed from previous steps.

Following the solution algorithm described in the previous section for the linear case, the following final results are obtained.

\subsection{The Order of Approximations}

The following final expressions can be used to obtain different order of approximations.

\section{a. The absolute value of zero order approximation is:}

$$
\left|u^{(0)}(t, z)\right|^{2}=\psi_{0}^{2}+\phi_{0}^{2}
$$

where

$$
\begin{aligned}
& \psi_{0}(t, z)=e^{-\gamma z} \sum_{n=0}^{\infty} T_{0 n}(z) \sin \left(\frac{n \pi}{T}\right) t \\
& \phi_{0}(t, z)=e^{-\gamma z} \sum_{n=0}^{\infty} \tau_{0 n}(z) \sin \left(\frac{n \pi}{T}\right) t \\
& G_{1}=-e^{\gamma z} F_{1}(t, z) \\
& G_{2}=e^{\gamma z} F_{2}(t, z)
\end{aligned}
$$

b. The absolute value of first order approximation is:

$$
\left|u^{(1)}(t, z)\right|^{2}=\left|u^{(0)}(t, z)\right|^{2}+2 \varepsilon\left(\psi_{0} \psi_{1}+\phi_{0} \phi_{1}\right)+\varepsilon^{2}\left(\psi_{1}{ }^{2}+\phi_{1}{ }^{2}\right)
$$

where 


$$
\begin{aligned}
& \psi_{1}(t, z)=e^{-\gamma z} \sum_{n=0}^{\infty} T_{1 n}(z) \sin \left(\frac{n \pi}{T}\right) t \\
& \phi_{1}(t, z)=e^{-\gamma z} \sum_{n=0}^{\infty} \tau_{1 n}(z) \sin \left(\frac{n \pi}{T}\right) t \\
& G_{1}=e^{-2 \gamma z}\left(\psi_{0}{ }^{3}+\psi_{0}{\phi_{0}}^{2}\right) \\
& G_{2}=e^{-2 \gamma z}\left(-\phi_{0}{ }^{3}-\phi_{0} \psi_{0}{ }^{2}\right)
\end{aligned}
$$

\section{c. The absolute value of second order approximation is:}

$$
\begin{aligned}
\left|u^{(2)}(t, z)\right|^{2}= & \left|u^{(1)}(t, z)\right|^{2}+2 \varepsilon^{2}\left(\psi_{0} \psi_{2}+\phi_{0} \phi_{2}\right)+2 \varepsilon^{3}\left(\psi_{1} \psi_{2}+\phi_{1} \phi_{2}\right) \\
& +\varepsilon^{4}\left(\psi_{2}{ }^{2}+\phi_{2}{ }^{2}\right)
\end{aligned}
$$

where

$$
\begin{aligned}
& \psi_{2}(t, z)=e^{-\gamma z} \sum_{n=0}^{\infty} T_{2 n}(z) \sin \left(\frac{n \pi}{T}\right) t \\
& \phi_{2}(t, z)=e^{-\gamma z} \sum_{n=0}^{\infty} \tau_{2 n}(z) \sin \left(\frac{n \pi}{T}\right) t \\
& G_{1}=e^{-2 \gamma z}\left(3 \psi_{0}{ }^{2} \psi_{1}+2 \psi_{0} \phi_{0} \phi_{1}+\psi_{1} \phi_{0}{ }^{2}\right) \\
& G_{2}=e^{-2 \gamma z}\left(-3 \phi_{0}{ }^{2} \phi_{1}-2 \phi_{0} \psi_{0} \psi_{1}-\phi_{1} \psi_{0}{ }^{2}\right)
\end{aligned}
$$

\section{Picard Approximation}

To validate the previous results, in the absence of the exact solution, let us follow another approximation technique. The Picard approximation is considered in this section.

Solving equation (25) with the same conditions (2) and (3) and following the Picard algorithm, which means that we solve the linear case iteratively [24]. This means that equation (25) can be rewritten as:

$$
\begin{gathered}
i \frac{\partial u(t, z)}{\partial z}+\alpha \frac{\partial^{2} u(t, z)}{\partial t^{2}}+i \gamma u(t, z)=-\varepsilon|u(t, z)|^{2} u(t, z)+F_{1}(t, z)+i F_{2}(t, z), \\
(t, z) \in(0, T) x(0, \infty)
\end{gathered}
$$

Let $u(t, z)=e^{-\gamma z}(\psi(t, z)+i \phi(t, z)), \psi, \phi$ : are real valued functions. The following coupled equations are got:

$\frac{\partial \phi(t, z)}{\partial z}=\alpha \frac{\partial^{2} \psi(t, z)}{\partial t^{2}}+\varepsilon\left(\psi^{2}+\phi^{2}\right) \psi-\gamma \phi-F_{1}(t, z)$ 
$\frac{\partial \psi(t, z)}{\partial z}=-\alpha \frac{\partial^{2} \phi(t, z)}{\partial t^{2}}-\varepsilon\left(\psi^{2}+\phi^{2}\right) \phi-\gamma \phi+F_{2}(t, z)$

where $\psi(t, 0)=f_{1}(t), \phi(t, 0)=f_{2}(t)$, and all other corresponding initial and boundary conditions are zeros.

\subsection{The order of approximations}

1. The absolute value of Zero order approximation is similar to perturbation method.

2. The absolute value of First order approximation:

$$
\begin{gathered}
i \frac{\partial u_{1}(t, z)}{\partial z}+\alpha \frac{\partial^{2} u_{1}(t, z)}{\partial t^{2}}+i \gamma u_{1}(t, z)=-\varepsilon\left|u_{0}(t, z)\right|^{2} u_{0}(t, z)+F_{1}(t, z)+i F_{2}(t, z), \\
(t, z) \in(0, T) x(0, \infty)
\end{gathered}
$$

with initial conditions $u_{1}(t, 0)=f_{1}(t)+i f_{2}(t)$ and boundary conditions

$u_{1}(0, z)=u_{1}(T, z)=0$.

$$
\left|u^{(1)}(t, z)\right|^{2}=\psi_{1}^{2}+\phi_{1}^{2}
$$

$\psi_{1}(t, z)=e^{-\gamma z} \sum_{n=0}^{\infty} T_{1 n}(z) \sin \left(\frac{n \pi}{T}\right) t$

$\phi_{1}(t, z)=e^{-\gamma z} \sum_{n=0}^{\infty} \tau_{1 n}(z) \sin \left(\frac{n \pi}{T}\right) t$

$G_{1}=-e^{\gamma z} F_{1}(t, z)+e^{-2 \gamma z} \varepsilon\left(\psi_{0}^{3}+\psi_{0} \phi_{0}^{2}\right)$

$G_{2}=e^{\gamma z} F_{2}(t, z)-e^{-2 \gamma z} \varepsilon\left(\phi_{0}{ }^{3}+\phi_{0} \psi_{0}^{2}\right)$

3. The absolute value of Second order approximation :

$$
\begin{gathered}
i \frac{\partial u_{2}(t, z)}{\partial z}+\alpha \frac{\partial^{2} u_{2}(t, z)}{\partial t^{2}}+i \gamma u_{2}(t, z)=-\varepsilon\left|u_{1}(t, z)\right|^{2} u_{1}(t, z)+F_{1}(t, z)+i F_{2}(t, z), \\
(t, z) \in(0, T) x(0, \infty)
\end{gathered}
$$

with initial conditions $u_{2}(t, 0)=f_{1}(t)+i f_{2}(t)$ and boundary conditions $u_{2}(0, z)=u_{2}(T, z)=0$.

$\left|u^{(2)}(t, z)\right|^{2}=\psi_{2}{ }^{2}+\phi_{2}{ }^{2}$

$\psi_{2}(t, z)=e^{-\gamma z} \sum_{n=0}^{\infty} T_{2 n}(z) \sin \left(\frac{n \pi}{T}\right) t$

$\phi_{2}(t, z)=e^{-\gamma z} \sum_{n=0}^{\infty} \tau_{2 n}(z) \sin \left(\frac{n \pi}{T}\right) t$ 


$$
\begin{aligned}
& G_{1}=-e^{\gamma z} F_{1}(t, z)+e^{-2 \gamma z} \varepsilon\left(\psi_{1}{ }^{3}+\psi_{1} \phi_{1}{ }^{2}\right) \\
& G_{2}=e^{\gamma z} F_{2}(t, z)-e^{-2 \gamma z} \varepsilon\left(\phi_{1}{ }^{3}+\phi_{1}{\psi_{1}}^{2}\right)
\end{aligned}
$$

\section{Case Studies}

To examine the proposed solution algorithm, we calculated many cases at different conditions of non-homogeneous term and initial conditions too.

\subsection{Perturbation Method:}

We illustrate here some cases of the case studies (Fig. 1 - Fig. 4)

Taking the case $F_{1}(t, z)=\rho_{1}, F_{2}(t, z)=0$ and $f_{1}(t)=\rho_{1} e^{-t}, f_{2}(t)=0$, the following selective results for the first and second order approximations are got:

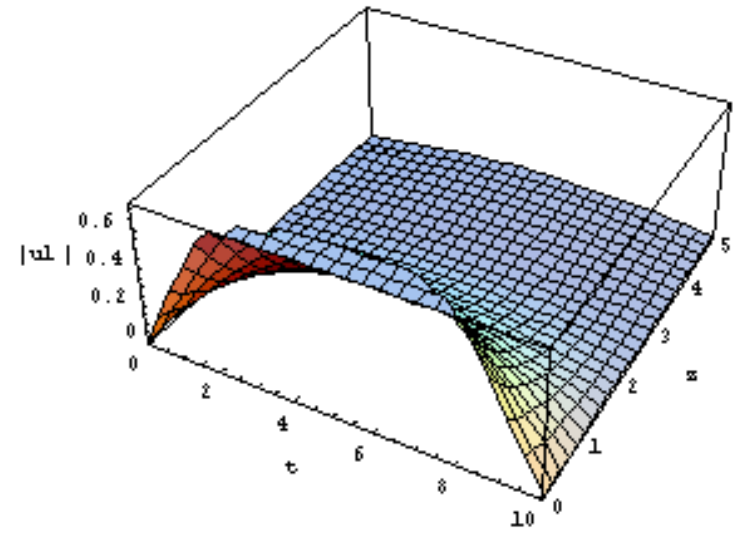

Fig. 1 The first order approximation of $\left|u^{(1)}\right|$ at $\varepsilon=1, \gamma=1$ and $\alpha, \rho_{1}, \rho_{2}=1, T=10$ with considering only one term on the series $(M=1)$.

Taking the case $F_{1}(t, z)=\rho_{1} \sin \left(\frac{m \pi}{T}\right) t, F_{2}(t, z)=0$ and $f_{1}(t)=\rho_{1} e^{-t}, f_{2}(t)=0$, the following selective results for the first and second order approximations are got:

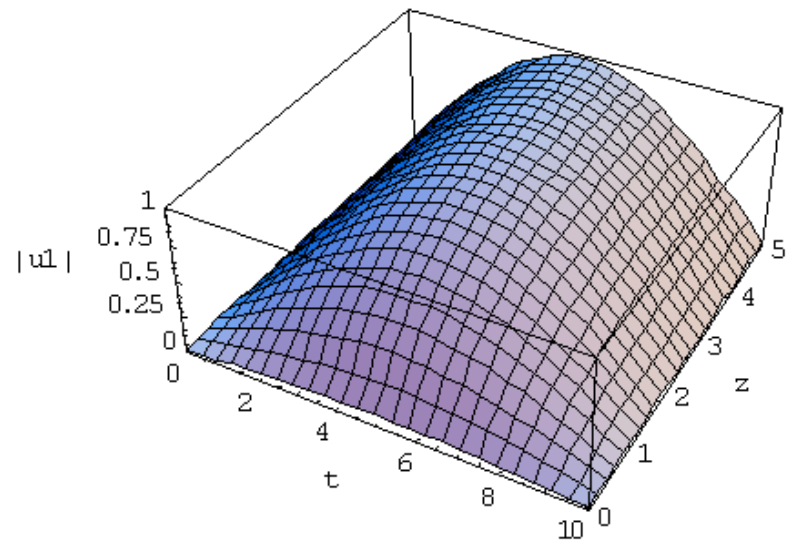

Fig. 2 The first order approximation of $\left|u^{(1)}\right|$ at $\varepsilon=0.2, \gamma=1$ and $\alpha, \rho_{1}, \rho_{2}=1, T=10$ with considering only one term on the series $(M=1)$.

Taking the case $F_{1}(t, z)=\rho_{1}, F_{2}(t, z)=0$ and $f_{1}(t)=\rho_{1} \sin \left(\frac{m \pi}{T}\right) t, f_{2}(t)=0$, the following selective results for the first and second order approximations are got: 


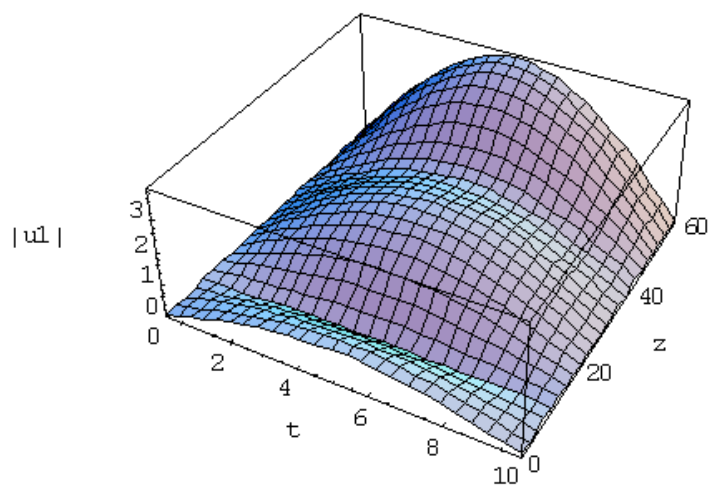

Fig. 3 The first order approximation of $\left|u^{(1)}\right|$ at $\varepsilon=0.2, \gamma=0$ and $\alpha, \rho_{1}, \rho_{2}=1, T=10$ with considering only one term on the series $(M=1)$.

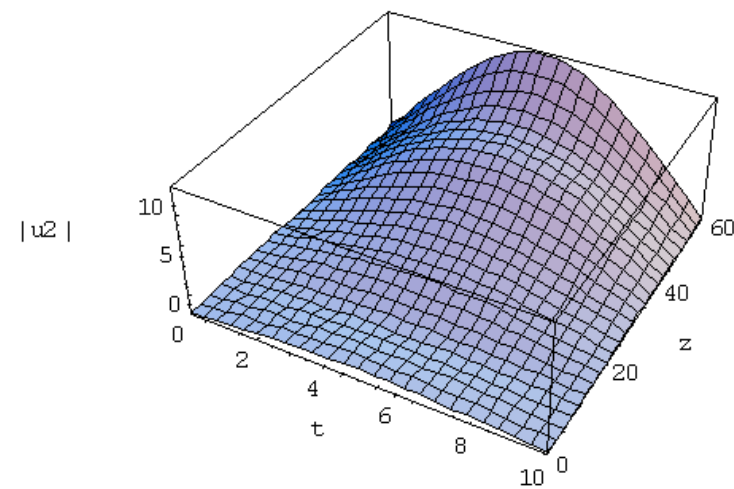

Fig. 4 The second order approximation of $\left|u^{(2)}\right|$ at $\varepsilon=0.2, \gamma=0$ and $\alpha, \rho_{1}, \rho_{2}=1, T=10$ with considering only ten terms on the series $(M=10)$.

Note: a lot of other case studies had been studied with combinations between constant, sinusoidal and exponential functions for both non-homogenous and initial conditions.

\subsection{Picard Approximation}

We illustrate here some of the case studies (Fig. 5 - Fig. 8).

Taking the case $F_{1}(t, z)=\rho_{1}, F_{2}(t, z)=0$ and $f_{1}(t)=\rho_{1}, f_{2}(t)=0$, the following selective results for the first and second order approximations are got:

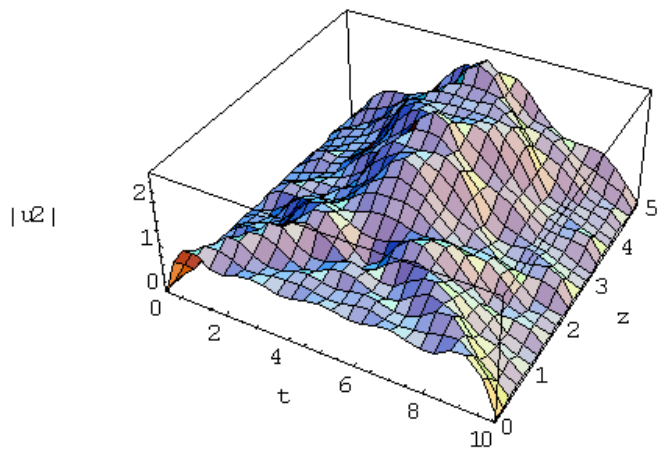

Fig. 5 The second order approximation of $\left|u^{(2)}\right|$ at $\varepsilon=0.2, \gamma=0$ and $\alpha, \rho_{1}, \rho_{2}=1, T=10$ with considering only one term on the series $(\mathrm{M}=10)$. 
Taking the case $F_{1}(t, z)=\rho_{1} e^{-t}, F_{2}(t, z)=0$ and $f_{1}(t)=\rho_{1} e^{-t}, f_{2}(t)=0$, the following selective results for the first and second order approximations are got:

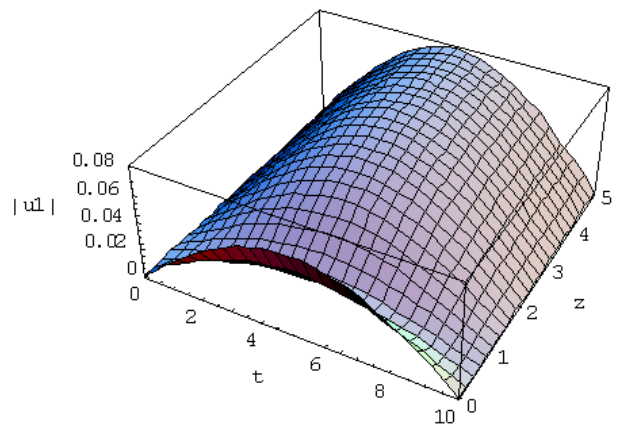

Fig. 6 The first order approximation of $\left|u^{(1)}\right|$ at $\varepsilon=1, \gamma=1$ and $\alpha, \rho_{1}, \rho_{2}=1, T=10$ with considering only one term on the series $(M=1)$.

Taking the case $F_{1}(t, z)=\rho_{1}, F_{2}(t, z)=0$ and $f_{1}(t)=\rho_{1} \sin \left(\frac{m \pi}{T}\right) t, f_{2}(t)=0$, the following selective results for the first and second order approximations are got:

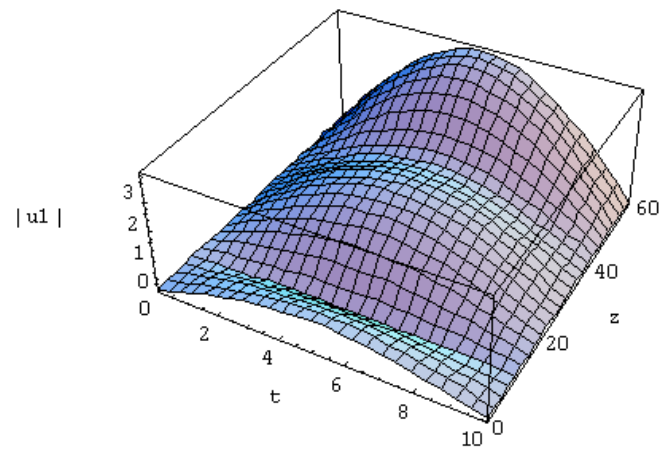

Fig. 7 The first order approximation of $\left|u^{(1)}\right|$ at $\varepsilon=0.2, \gamma=0$ and $\alpha, \rho_{1}, \rho_{2}=1, T=10$ with considering only one term on the series $(M=1)$.

Taking the case $F_{1}(t, z)=\rho_{1} e^{-t}, F_{2}(t, z)=0$ and $f_{1}(t)=\rho_{1} \sin \left(\frac{m \pi}{T}\right) t, f_{2}(t)=0$, the following selective results for the first and second order approximations are got:

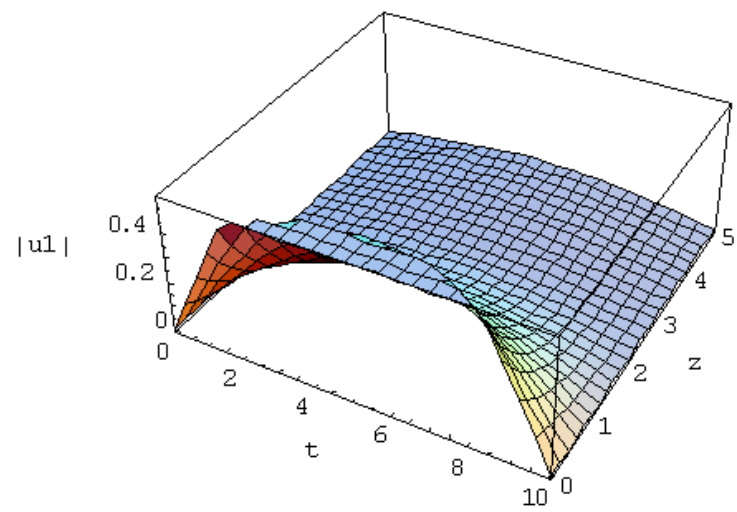

Fig. 8 The first order approximation of $\left|u^{(1)}\right|$ at $\varepsilon=0.2, \gamma=1$ and $\alpha, \rho_{1}, \rho_{2}=1, T=10$ with considering only ten terms on the series $(M=1)$. 
Note: a lot of other case studies had been studied with combinations between constant, sinusoidal and exponential functions for both non-homogenous and initial conditions.

\section{Comparison between Perturbation \& Picard Approximation}

We are here give both perturbation and Picard results in same graph for some selected cases to compare between two methods, (Fig. 9 - Fig. 11)

Taking the case $F_{1}(t, z)=\rho_{1}, F_{2}(t, z)=0$ and $f_{1}(t)=\rho_{1}, f_{2}(t)=0$.

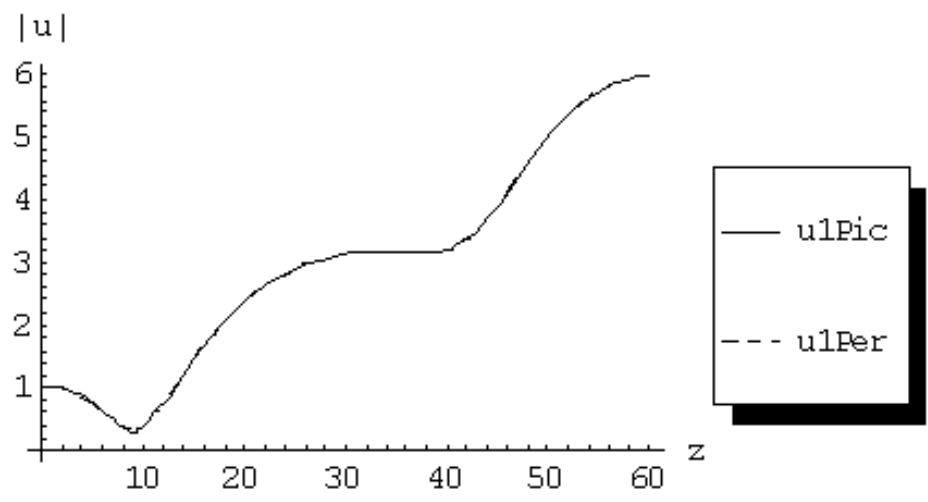

Fig. 9 Comparison between Picard approximation and Perturbation method for first order at $\varepsilon=0.2, \gamma=0$ and $\alpha, \rho_{1}, \rho_{2}=1, T=10, t=3$.

Taking the case $F_{1}(t, z)=\rho_{1} e^{-t}, F_{2}(t, z)=0$ and $f_{1}(t)=\rho_{1} e^{-t}, f_{2}(t)=0$.

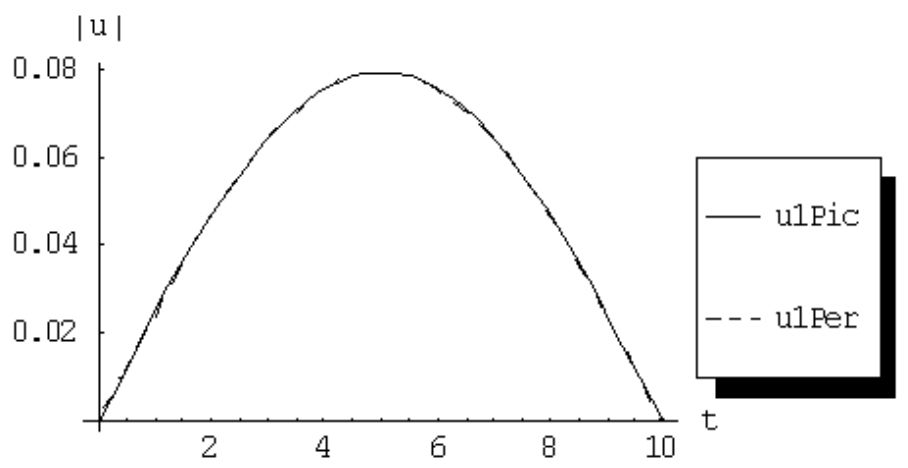

Fig. 10 Comparison between Picard approximation and Perturbation method for first order at $\varepsilon=0.2, \gamma=1$ and $\alpha, \rho_{1}, \rho_{2}=1, T=10, z=5$.

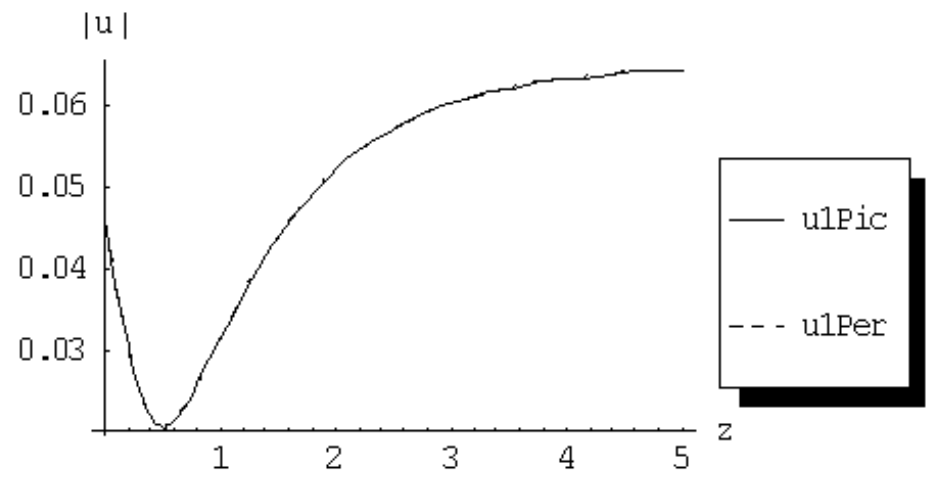

Fig. 11 Comparison between Picard approximation and Perturbation method for first order at $\varepsilon=0.2, \gamma=1$ and $\alpha, \rho_{1}, \rho_{2}=1, T=10, t=3$. 


\section{T Study}

We are here examining the behavior of Perturbation method and Picard Approximation against different values of $\mathrm{T}$ through case studies on the same graph.

\subsection{Perturbation Method}

We are here illustrating the effect of the change of the time interval $\mathrm{T}$ on the solution and showing that through many case studies. Some of them are summarized through (Fig. 12 Fig. 14)

Taking the case $F_{1}(t, z)=\rho_{1}, F_{2}(t, z)=0, f_{1}(t)=\rho_{1}, f_{2}(t)=0$, the following selective results for the first and second order approximations are got:

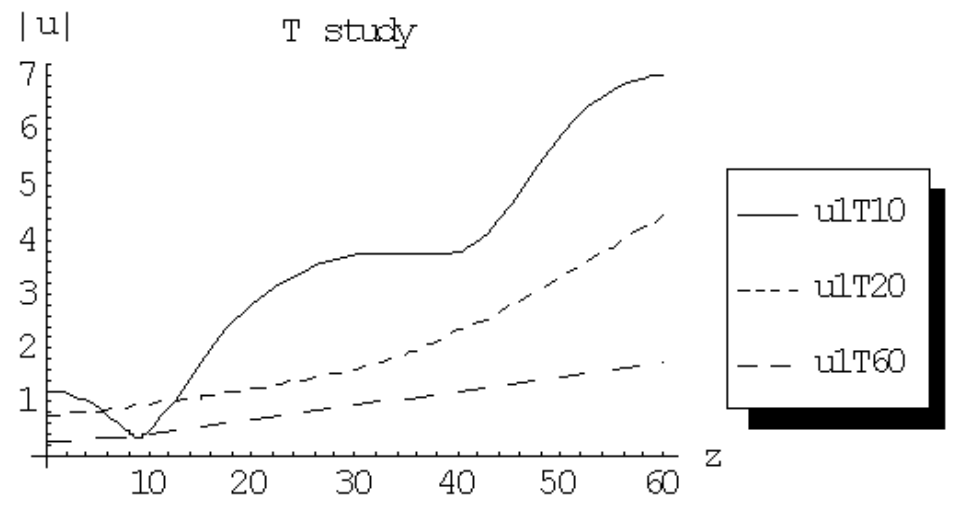

Fig. 12 The first order approximation of $\left|u^{(1)}\right|$ at $\varepsilon=0.2, \gamma=0$ and $\alpha, \rho_{1}, \rho_{2}=1, M=10, t=4$ for different values of $T=10,20$ and 60 respectively.

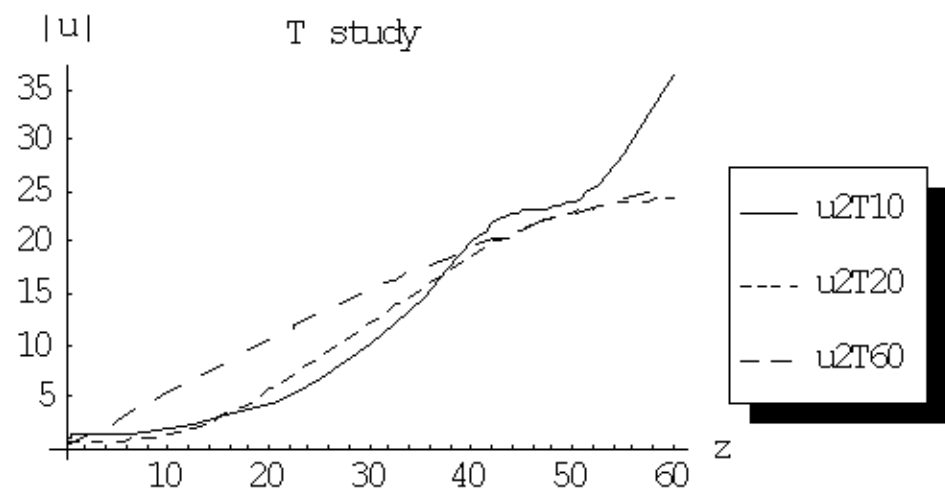

Fig. 13 The second order approximation of $\left|u^{(2)}\right|$ at $\varepsilon=0.2, \gamma=0$ and $\alpha, \rho_{1}, \rho_{2}=1, M=10, t=4$ for different values of $T=10,20$ and 60 respectively.

Taking the case $F_{1}(t, z)=\rho_{1} e^{-t}, F_{2}(t, z)=0, f_{1}(t)=\rho_{1} \sin \left(\frac{m \pi}{T} t\right), f_{2}(t)=0$, the following selective results for the first and approximation are got: 


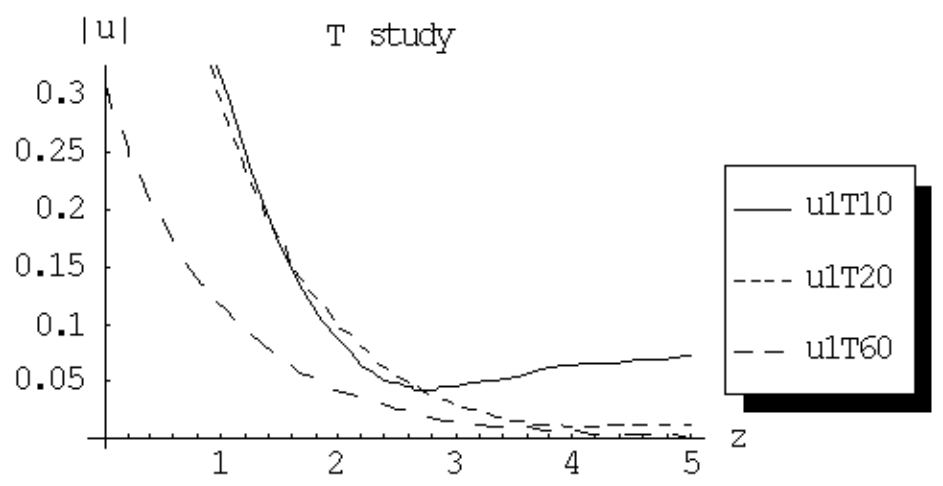

Fig. 14 The first order approximation of $\left|u^{(1)}\right|$ at $\varepsilon=0.2, \gamma=1$ and $\alpha, \rho_{1}, \rho_{2}=1, M=1, t=6$ for different values of $T=10,20$ and 60 respectively.

It is clear from case studies that as $T$ increases from $T=10, T=20, T=60$ the magnitude of $u(t, z)$ decreases accordingly.

\subsection{Picard Approximation}

We are here to study the effect of change of the time interval $\mathrm{T}$ on the solution through many case studies. Some of them are illustrated (Fig. 15 - Fig. 16).

Taking the case $F_{1}(t, z)=\rho_{1}, F_{2}(t, z)=0, f_{1}(t)=\rho_{1}, f_{2}(t)=0$ and following the algorithm, the following selective results for the first and second order approximations are got:

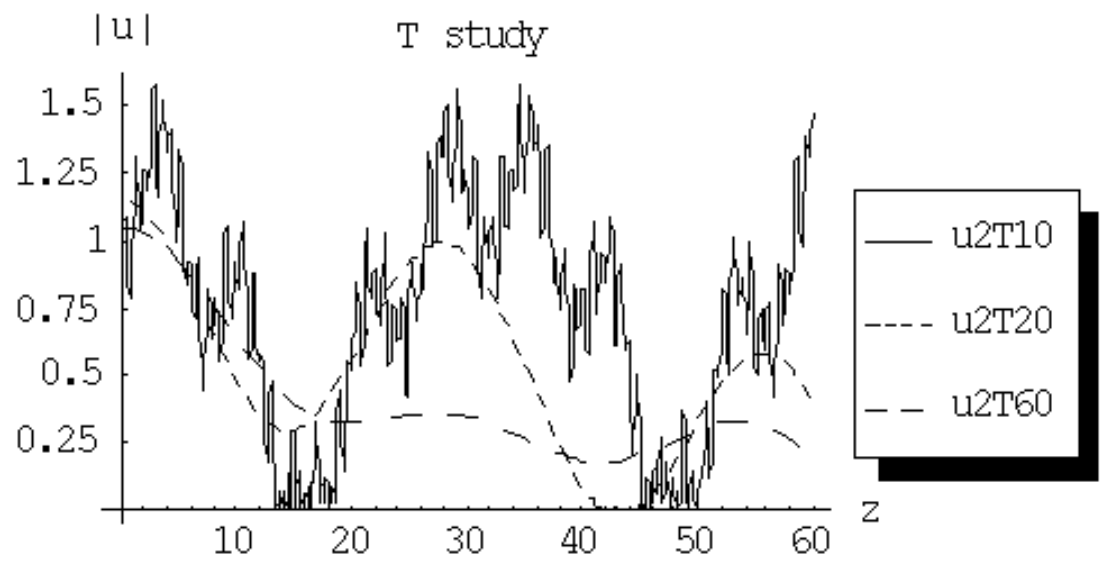

Fig. 15 The second order approximation of $\left|u^{(2)}\right|$ at $\varepsilon=0.002, \gamma=0$ and $\alpha, \rho_{1}, \rho_{2}=1, M=10, t=4$ for different values of $T=10,20$ and 60 respectively.

Taking the case $\quad F_{1}(t, z)=\rho_{1} \sin \left(\frac{m \pi}{T} t\right), F_{2}(t, z)=0, f_{1}(t)=\rho_{1} e^{-t}, f_{2}(t)=0$ and following the algorithm, the following selective results for the first approximation are got: 


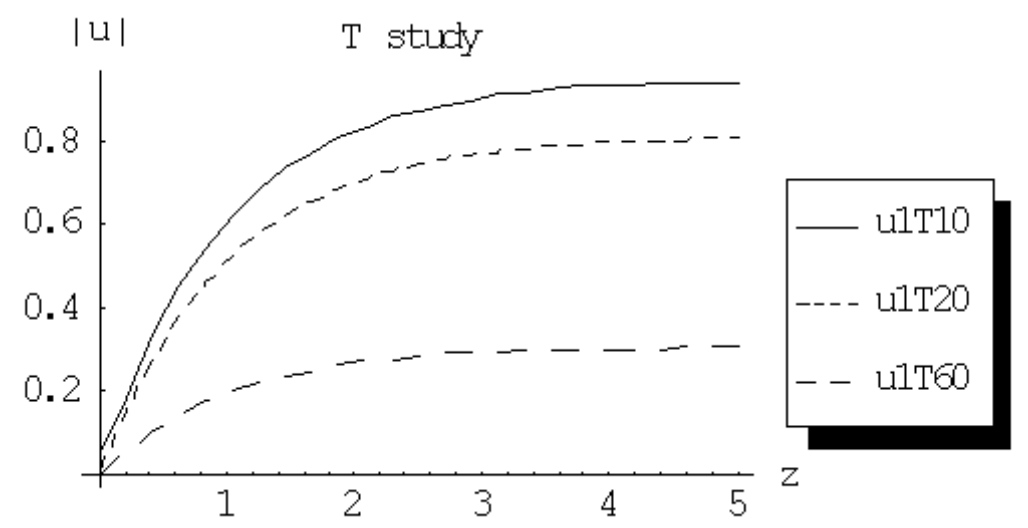

Fig. 16 The first order approximation of $\left|u^{(1)}\right|$ at $\varepsilon=0.2, \gamma=1$ and $\alpha, \rho_{1}, \rho_{2}=1, M=1, t=6$ for different values of $T=10,20$ and 60 respectively.

It is clear from case studies that as $T$ increases from $T=10, T=20, T=60$ the magnitude of $u(t, z)$ decreases accordingly.

\section{Conclusions}

The stability of the solution of the cubic nonlinear non-homogeneous Schrodinger equation is highly affected in the absence of gamma. The perturbation as well as the Picard methods introduce approximate solutions for such problems where second or third order of approximations can be obtained from which some parametric studies can be achieved to illustrate the solution behavior under the change of the problem physical parameters. The use of Mathematica, or any other symbolic code, makes the use of the solution algorithm possible and can develop a solution procedure which can help in getting some knowledge about the solution.

\section{References}

[1] Cazenave T. and Lions P., "Orbital stability of standing waves for some nonlinear Schrodinger equations", Commun. Math. Phys. 85(1982) 549-561.

[2] Faris W.G. and Tsay W.J., "Time delay in random scattering", SIAM J. on applied mathematics 54(2) (1994) 443-455.

[3] Bruneau C., Menza L., and Lehner T., "Numerical resolution of some nonlinear Schrodinger-like equations in plasmas", Numer. Math. PDEs 15(6) (1999) 672-696.

[4] Abdullaev F. and Granier J., "Solitons in media with random dispersive perturbations", Physica (D)134(1999) 303-315.

[5] Corney J.F. and Drummond P., "Quantum noise in Optical fibers.II.Raman Jitter in soliton communications, J. Opt.Soc.Am. B 18(2)(2001) 153-161.

[6] Biswas A. and Milovic D., "Bright and dark solitons of generalized Schrodiner equation", Communications in nonlinear Science and Numerical Simulation, DOI: 10.1016/j.cnsns.2009.06.017

[7] Staliunas K., "Vortices and dark solitons in the two-dimensional nonlinear Schrodinger equation", Chaos, Solitons and Fractals 4(1994) 1783-1796. 
[8] Carretero R., Talley J.D., Chong C. and Malomed B.A., "Multistable solitons in the cubic-quintic discrete nonlinear Schrodinger equation", Physics letter A 216(2006) 7789.

[9] Seenuvasakumaran P., Mahalingam A. and Porsezian K., "Dark solitons in N-coupled higher order nonlinear Schrodinger equations", Communications in Nonlinear science and Numerical simulation 13(2008) 1318-1328.

[10] Xing Lü, Bo Tian, Tao Xu, Ke-Jie Cai and Wen-Jun Liu, "Analytical study of nonlinear Schrodinger equation with an arbitrary linear-time potential in quasi one dimensional Bose-Einstein condensates", Annals of Physics 323(2008) 2554-2565.

[11] Debussche A. and Menza L., "Numerical simulation of focusing stochastic nonlinear Schrodinger equations", Physica D 162(2002) 131-154.

[12] Debussche A. and Menza L., "Numerical resolution of stochastic focusing NLS equations", Applied mathematics letters 15(2002) 661-669.

[13] M. Wang, X. Li, and J. Zhang, "Various exact solutions of nonlinear Schrödinger equation with two nonlinear terms", Chaos, Solitons and Fractals, vol. 31, no. 3, pp. 594-601, 2007.

[14] Xu L. and Zhang J., "Exact solutions to two higher order nonlinear Schrodinger equations", Chaos, Solitons and Fractals 31(2007) 937-942.

[15] Sweilam N., "Variational iteration method for solving cubic nonlinear Schrodinger equation", J. of computational and applied mathematics 207(2007) 155-163.

[16] Zhu S., "Exact solutions for the high order dispersive cubic-quintic nonlinear Schrodinger equation by the extended hyperbolic auxiliary equation method", Chaos, Solitons and Fractals (2006) 960-779.

[17] Sun J., Qi Ma Z., Hua W. and Zhao Qin M., "New conservation schemes for the nonlinear Schrodinger equation", Applied mathematics and computation 177(2006) 446-451.

[18] Porsezian K. and Kalithasan B., "Cnoidal and solitary wave solutions of the coupled higher order nonlinear Schrodinger equations in nonlinear optics", Chaos, Solitons and Fractals 31(2007) 188-196.

[19] Sakaguchi H. and Higashiuchi T., "Two-dimensional dark soliton in the nonlinear Schrodinger equation", Physics letters A 39(2006) 647-651.

[20] Huang D., Li D.S. and Zhang H., "Explicit and exact traveling wave solution for the generalized derivative Schrodinger equation", Chaos, Solitons and Fractals 31(3) (2007) 586-593.

[21] Magdy A. El-Tawil, H. El Zoheiry and Sherif E. Nasr "Nonlinear Cubic Homogenous Schrodinger Equations with Complex Initial Conditions, Limited Time Response", The Open Applied mathematics Journal, 4(2010) 6-17

[22] El-Tawil A., El-Hazmy A., "Perturbative nonlinear Schrodinger equations under variable group velocity dissipation", Far East Journal math. Sciences, 27(2) (2007) 419 $-430$.

[23] El-Tawil A., El Hazmy A., "On perturbative cubic nonlinear Schrodinger equations under complex non-homogeneities and complex initial conditions", Journal Differential Equations and Nonlinear Mechanics, DOI: 10.1155/2009/395894

[24] Pipes L. and Harvill L., "Applied Mathematics for Engineers and Physicists", McGraw -Hill, Tokyo, 1970. 